\title{
MODULATION OF NONLINEAR WAVES IN A FLUID-FILLED ELASTIC TUBE WITH STENOSIS
}

\author{
HILMI DEMIRAY
}

Received 23 June 2004

\begin{abstract}
Treating the arteries as a thin-walled and prestressed elastic tube with a stenosis and the blood as a Newtonian fluid with negligible viscosity, we have studied the amplitude modulation of nonlinear waves in such a composite system by use of the reductive perturbation method. The governing evolution equation is obtained as the variable-coefficient nonlinear Schrödinger equation. It is observed that the speed of the harmonic wave increases with distance from the center of stenosis.
\end{abstract}

2000 Mathematics Subject Classification: 74F10.

1. Introduction. The striking feature of the arterial blood flow is its pulsatile character. The intermittent ejection of blood from the left ventricle produces pressure and flow pulses in the arterial tree. Experimental studies reveal that flow velocity in blood vessels largely depends on the elastic properties of the vessel wall and they propagate towards the periphery with a characteristic pattern [11].

Due to its applications in arterial mechanics, the propagation of pressure pulses in fluid-filled distensible tubes has been studied by several researchers (Pedley [12] and Fung [8]). Most of the works on wave propagation in compliant tubes have considered small amplitude waves ignoring the nonlinear effects and focused on the dispersive character of waves (see Atabek and Lew [2], Rachev [13], and Demiray [5]). However, when the nonlinear terms arising from the constitutive equations and kinematical relations are introduced, one has to consider either finite amplitude or small-but-finite amplitude waves, depending on the order of nonlinearity.

The propagation of finite amplitude waves in fluid-filled elastic or viscoelastic tubes has been examined, for instance, by Rudinger [15], Anliker et al. [1], and Tait and Moodie [17] by using the method of characteristics in studying the shock formation. On the other hand, the propagation of small-but-finite amplitude waves in distensible tubes has been investigated by Johnson [10], Hashizume [9], Yomosa [18], and Demiray [6] by employing various asymptotic methods. In all these works [6, 9, 10, 18], depending on the balance between the nonlinearity, dispersion and dissipation, the Korteweg-de Vries (KdV), Burgers or KdV-Burger equations are obtained as the evolution equations.

As is well known, when the nonlinear effects are small, the system of equations that describe the physical phenomenon admits harmonic wave solution with constant amplitude. If the amplitude of the wave is small-but-finite, the nonlinear terms cannot be neglected and the nonlinearity gives rise to the variation of amplitude in both 
space and time variables. When the amplitude varies slowly over a period of oscillation, a stretching transformation allows us to decompose the system into a rapidly varying part associated with the oscillation and a slowly varying part such as the amplitude. A formal solution can be given in the form of an asymptotic expansion, and an equation determining the modulation of the first-order amplitude can be derived. For instance, the nonlinear Schrödinger (NLS) equation is the simplest representative equation describing the self-modulation of one-dimensional monochromatic plane waves in dispersive media. It exhibits a balance between the nonlinearity and dispersion. The problem of self-modulation of small-but-finite amplitude waves in fluid-filled distensible tubes was considered by Ravindran and Prasad in [14], in which they showed that for a linear elastic tube wall model, the nonlinear self-modulation of pressure waves is governed by the NLS equation. Demiray [7], employing the exact equations of a viscous fluid and of a prestressed elastic tube, studied the amplitude modulation of nonlinear waves and obtained the dissipative (NLS) equation as the governing equation. In all these works, the arteries are considered as a cylindrical tube with constant radius. In essence, the radii of the arteries are variable along the axis of the tube.

In the present work, treating the arteries as a thin-walled and prestressed elastic tube with a stenosis and the blood as a Newtonian fluid with negligible viscosity, we have studied the amplitude modulation of nonlinear waves in such a composite system by use of the reductive perturbation method. For that purpose, we listed the basic equations governing the radial motion of the tube and of the fluid body in Section 2 . Considering the problem that we are concerned with to be of initial-value type, we introduced a set of stretched coordinates and obtained the equations of perturbed field in Section 3 and gave their solution in Section 4. The governing evolution equation is obtained as the variable-coefficient nonlinear Schrödinger equation. By seeking a progressive wave type of solution to the variable-coefficient NLS equation, it is found that the speed of the harmonic wave increases with distance from the center of stenosis. Such a result is to be expected from physical considerations.

\section{Basic equations and theoretical preliminaries}

2.1. Equations of tube. In this section, we will derive the basic equations governing the motion of a prestressed thin elastic tube, with an axially symmetric bump (stenosis), and filled with a viscous fluid. For that purpose, we consider a circularly cylindrical tube of radius $R_{0}$. It is assumed that such a tube is subjected to an axial stretch $\lambda_{z}$ and static inner pressure $P_{0}^{*}(Z)$. Under the effect of such a variable pressure, the position vector of a generic point on the tube is assumed to be described by

$$
\mathbf{r}_{0}=\left[r_{0}-f^{*}\left(z^{*}\right)\right] \mathbf{e}_{\mathbf{r}}+z^{*} \mathbf{e}_{\mathbf{z}}, \quad z^{*}=\lambda_{z} Z,
$$

where $\mathbf{e}_{\mathbf{r}}, \mathbf{e}_{\theta}$, and $\mathbf{e}_{\mathbf{z}}$ are the unit base vectors in the cylindrical polar coordinates, $\boldsymbol{r}_{0}$ is the deformed radius at the origin of the coordinate system, $Z$ is the axial coordinate before the deformation, $z^{*}$ is the axial coordinate after static deformation, and $f^{*}\left(z^{*}\right)$ 
is a function that characterizes the axially symmetric bump on the surface of the arterial wall and will be specified later.

Upon this initial static deformation, we will superimpose a dynamical radial displacement $u^{*}\left(z^{*}, t^{*}\right)$, where $t^{*}$ is the time parameter, but, in view of the external tethering, the axial displacement is assumed to be negligible. Then, the position vector $\mathbf{r}$ of a generic point on the tube may be described by

$$
\mathbf{r}=\left[r_{0}-f^{*}\left(z^{*}\right)+u^{*}\right] \mathbf{e}_{\mathbf{r}}+z^{*} \mathbf{e}_{\mathbf{z}}
$$

The arc lengths along the meridional and circumferential curves are, respectively, given by

$$
d s_{z}=\left[1+\left(-f^{*^{\prime}}+\frac{\partial u^{*}}{\partial z^{*}}\right)^{2}\right]^{1 / 2} d z^{*}, \quad d s_{\theta}=\left(r_{0}-f^{*}+u^{*}\right) d \theta
$$

Then, the stretch ratios along the meridional and circumferential curves, respectively, may be given by

$$
\lambda_{1}=\lambda_{z}\left[1+\left(-f^{*^{\prime}}+\frac{\partial u^{*}}{\partial z^{*}}\right)^{2}\right]^{1 / 2}, \quad \lambda_{2}=\frac{1}{R_{0}}\left(r_{0}-f^{*}+u^{*}\right),
$$

where the prime denotes the differentiation of the corresponding quantity with respect to $z^{*}$. The unit tangent vector $\mathbf{t}$ along the deformed meridional curve and the unit exterior normal vector $\mathbf{n}$ to the deformed tube are given by

$$
\mathbf{t}=\frac{\left(-f^{*^{\prime}}+\partial u^{*} / \partial z^{*}\right) \mathbf{e}_{\mathbf{r}}+\mathbf{e}_{\mathbf{z}}}{\left[1+\left(-f^{*^{\prime}}+\partial u^{*} / \partial z^{*}\right)^{2}\right]^{1 / 2}}, \quad \mathbf{n}=\frac{\mathbf{e}_{\mathbf{r}}-\left(-f^{*^{\prime}}+\partial u^{*} / \partial z^{*}\right) \mathbf{e}_{\mathbf{z}}}{\left[1+\left(-f^{*^{\prime}}+\partial u^{*} / \partial z^{*}\right)^{2}\right]^{1 / 2}}
$$

Let $T_{1}$ and $T_{2}$ be the membrane forces along the meridional and circumferential curves, respectively. Then, the equation of the radial motion of a small tube element placed between the planes $z^{*}=$ const, $z^{*}+d z^{*}=$ const, $\theta=$ const, and $\theta+d \theta=$ const may be given by

$$
\begin{aligned}
& -T_{2}\left[1+\left(-f^{*^{\prime}}+\frac{\partial u^{*}}{\partial z^{*}}\right)^{2}\right]^{1 / 2}+\frac{\partial}{\partial z^{*}}\left\{\frac{\left(r_{0}-f^{*}+u^{*}\right)\left(-f^{*^{\prime}}+\partial u^{*} / \partial z^{*}\right)}{\left[1+\left(f^{*^{\prime}}+\partial u^{*} / \partial z^{*}\right)^{2}\right]^{1 / 2}} T_{1}\right\} \\
& +P^{*}\left(r_{0}-f^{*}+u^{*}\right)=\rho_{0} \frac{H R_{0}}{\lambda_{z}} \frac{\partial^{2} u^{*}}{\partial t^{* 2}}
\end{aligned}
$$


where $\rho_{0}$ is the mass density of the tube, $H$ is the thickness in the undeformed configuration, and $P^{*}$ is inner pressure applied by the fluid.

Let $\mu \Sigma$ be the strain energy density function of the membrane, where $\mu$ is the shear modulus of the tube material. Then, the membrane forces may be expressed in terms of the stretch ratios as

$$
T_{1}=\frac{\mu H}{\lambda_{2}} \frac{\partial \Sigma}{\partial \lambda_{1}}, \quad T_{2}=\frac{\mu H}{\lambda_{1}} \frac{\partial \Sigma}{\partial \lambda_{2}} .
$$

Introducing (2.7) into (2.6), the equation of motion of the tube in the radial direction takes the following form:

$$
\begin{gathered}
-\frac{\mu}{\lambda_{z}} \frac{\partial \Sigma}{\partial \lambda_{2}}+\mu R_{0} \frac{\partial}{\partial z^{*}}\left\{\frac{\left(-f^{*^{\prime}}+\partial u^{*} / \partial z^{*}\right)}{\left[1+\left(-f^{*^{\prime}}+\partial u^{*} / \partial z^{*}\right)^{2}\right]^{1 / 2}} \frac{\partial \Sigma}{\partial \lambda_{1}}\right\} \\
+\frac{P^{*}}{H}\left(r_{0}-f^{*}+u^{*}\right)=\rho_{0} \frac{R_{0}}{\lambda_{z}} \frac{\partial^{2} u^{*}}{\partial t^{* 2}}
\end{gathered}
$$

2.2. Equations of fluid. In general, blood is known to be an incompressible nonNewtonian fluid. The main factor for blood to behave like a non-Newtonian fluid is the level of cell concentration (hematocrit ratio) and the deformability of red blood cells. In the course of blood flow in arteries, the red cells move to the central region of the artery and, thus, the hematocrit ratio is reduced near the arterial wall, where the shear rate is quite high, as can be seen from Poiseuille flow. Experimental studies indicate that when the hematocrit ratio is low and the shear rate is high, blood behaves like a Newtonian fluid (see [12]). Moreover, as pointed out by Rudinger [15], for flows in large blood vessels, the viscosity of blood may be neglected as a first approximation. For this case, the variation of the field quantities with the radial coordinate may be neglected. Thus, the averaged equations of motion of an incompressible fluid may be given by

$$
\begin{gathered}
\frac{\partial A^{*}}{\partial t^{*}}+\frac{\partial}{\partial z^{*}}\left(A^{*} w^{*}\right)=0, \\
\frac{\partial w^{*}}{\partial t^{*}}+w^{*} \frac{\partial w^{*}}{\partial z^{*}}+\frac{1}{\rho_{f}} \frac{\partial P^{*}}{\partial z^{*}}=0,
\end{gathered}
$$

where $A^{*}$ is the cross-sectional area of the tube, $w^{*}$ is the averaged axial fluid velocity, and $P^{*}$ is the averaged fluid pressure. Noting the relation between the cross-sectional area and the final radius, that is, $A^{*}=\pi\left(r_{0}-f^{*}+u^{*}\right)^{2}$, (2.9) reads

$$
2 \frac{\partial u^{*}}{\partial t^{*}}+2 w^{*} \frac{\partial u^{*}}{\partial z^{*}}+\left(r_{0}-f^{*}+u^{*}\right) \frac{\partial w^{*}}{\partial z^{*}}=0
$$


At this stage it is convenient to introduce the following nondimensionalized quantities:

$$
\begin{array}{lll}
t^{*}=\left(\frac{R_{0}}{c_{0}}\right) t, & z^{*}=R_{0} z, & u^{*}=R_{0} u, \\
m=\frac{\rho_{0} H}{\rho_{f} R_{0}}, & w^{*}=c_{0} w, & f^{*}=R_{0} f, \\
r_{0}=R_{0} \lambda_{\theta}, & P^{*}=\rho_{f} c_{0}^{2} p, & c_{0}^{2}=\frac{\mu H}{\rho_{f} R_{0}} .
\end{array}
$$

Introducing (2.12) into equations (2.8), (2.9), and (2.10), we obtain the following nondimensionalized equations:

$$
\begin{gathered}
2 \frac{\partial u}{\partial t}+2\left(-f^{\prime}+\frac{\partial u}{\partial z}\right) w+\left(\lambda_{\theta}-f+u\right) \frac{\partial w}{\partial z}=0, \\
\frac{\partial w}{\partial t}+w \frac{\partial w}{\partial z}+\frac{\partial p}{\partial z}=0, \\
p=\frac{m}{\lambda_{z}\left(\lambda_{\theta}-f+u\right)} \frac{\partial^{2} u}{\partial t^{2}}+\frac{1}{\lambda_{z}\left(\lambda_{\theta}-f+u\right)} \frac{\partial \Sigma}{\partial \lambda_{2}} \\
-\frac{1}{\left(\lambda_{\theta}-f+u\right)} \frac{\partial}{\partial z}\left\{\frac{\left(-f^{\prime}+\partial u / \partial z\right)}{\left[1+\left(-f^{\prime}+\partial u / \partial z\right)^{2}\right]^{1 / 2}} \frac{\partial \Sigma}{\partial \lambda_{1}}\right\} .
\end{gathered}
$$

For our future purposes we need the power series expansion of $p$ in terms of $\bar{u}=u-f$ and its temporal and spatial derivatives. If the expression (2.15) is expanded into a power series in $u$, up to and including the cubic terms, we obtain

$$
p=p_{0}+L_{1}(u)+L_{2}(u)+L_{3}(u)+\cdots,
$$

where $p_{0}$ is the constant ambient pressure and $L_{1}(u), L_{2}(u)$, and $L_{3}(u)$ are defined by

$$
\begin{aligned}
L_{1}(u)= & \frac{m}{\lambda_{\theta} \lambda_{z}} \frac{\partial^{2} \bar{u}}{\partial t^{2}}-\alpha_{0} \frac{\partial^{2} \bar{u}}{\partial z^{2}}+\beta_{1} \bar{u}, \quad p_{0}=\beta_{0}, \\
L_{2}(u)= & -\frac{m}{\lambda_{\theta}^{2} \lambda_{z}} \bar{u} \frac{\partial^{2} \bar{u}}{\partial t^{2}}-\alpha_{1}\left(\frac{\partial \bar{u}}{\partial z}\right)^{2}-\left(2 \alpha_{1}-\frac{\alpha_{0}}{\lambda_{\theta}}\right) \bar{u} \frac{\partial^{2} \bar{u}}{\partial z^{2}}+\beta_{2} \bar{u}^{2} \\
L_{3}(u)= & \frac{m}{\lambda_{\theta}^{3} \lambda_{z}} \bar{u}^{2} \frac{\partial^{2} \bar{u}}{\partial t^{2}}-\left(\alpha_{2}-\frac{\alpha_{1}}{\lambda_{\theta}}\right) \bar{u}\left(\frac{\partial \bar{u}}{\partial z}\right)^{2}-\left(\alpha_{2}-\frac{2 \alpha_{1}}{\lambda_{\theta}}+\frac{\alpha_{0}}{\lambda_{\theta}^{2}}\right) \bar{u}^{2} \frac{\partial^{2} \bar{u}}{\partial z^{2}} \\
& -3\left(\gamma_{1}-\frac{\alpha_{0}}{2}\right)\left(\frac{\partial \bar{u}}{\partial z}\right)^{2} \frac{\partial^{2} \bar{u}}{\partial z^{2}}+\beta_{3} \bar{u}^{3},
\end{aligned}
$$


where the coefficients $\alpha_{0}, \alpha_{2}, \beta_{0}, \ldots, \beta_{3}$, and $\gamma_{1}$ are defined by

$$
\begin{gathered}
\alpha_{0}=\frac{1}{\lambda_{\theta}} \frac{\partial \Sigma}{\partial \lambda_{z}}, \quad \alpha_{1}=\frac{1}{2 \lambda_{\theta}} \frac{\partial^{2} \Sigma}{\partial \lambda_{\theta} \lambda_{z}}, \quad \alpha_{2}=\frac{1}{2 \lambda_{\theta}} \frac{\partial^{3} \Sigma}{\partial \lambda_{\theta}^{2} \partial \lambda_{z}}, \\
\beta_{0}=\frac{1}{\lambda_{\theta} \lambda_{z}} \frac{\partial \Sigma}{\partial \lambda_{\theta}}, \quad \beta_{1}=\frac{1}{\lambda_{\theta} \lambda_{z}} \frac{\partial^{2} \Sigma}{\partial \lambda_{\theta}^{2}}-\frac{\beta_{0}}{\lambda_{\theta}}, \\
\beta_{2}=\frac{1}{2 \lambda_{\theta} \lambda_{z}} \frac{\partial^{3} \Sigma}{\partial \lambda_{\theta}^{2}}-\frac{\beta_{1}}{\lambda_{\theta}}, \quad \beta_{3}=\frac{1}{6 \lambda_{\theta} \lambda_{z}} \frac{\partial^{4} \Sigma}{\partial \lambda_{\theta}^{4}}-\frac{\beta_{2}}{\lambda_{\theta}}, \\
\gamma_{1}=\frac{\lambda_{z}}{2 \lambda_{\theta}} \frac{\partial^{2} \Sigma}{\partial \lambda_{z}^{2}} .
\end{gathered}
$$

These equations give sufficient relations to determine the field variables $u, w$, and $p$ completely.

3. Nonlinear wave modulation. In this section, we will examine the amplitude modulation of weakly nonlinear waves in a fluid-filled elastic tube with a stenosis, whose dimensionless governing equations are given in (2.13), (2.14), and (2.16). Considering the dispersion relation of the linearized field equations and the nature of the problem of concern, which is an initial value problem, the following stretched coordinates may be introduced:

$$
\xi=\epsilon(z-\lambda t), \quad \tau=\epsilon^{2} z
$$

where $\epsilon$ is a smallness parameter measuring the weakness of nonlinearity and $\lambda$ is a constant to be determined from the solution. Solving $z$ in terms of the variable $\tau$, we get $z=\tau / \epsilon^{2}$. Introducing this expression of $z$ into the expression of $f(z)$, we obtain

$$
f(z)=h(\epsilon, \tau) .
$$

Here, we will assume that $h(\epsilon, \tau)$ is only the function of the slow variable $\tau$ and the smallness parameter $\epsilon$. Furthermore, in order to take the effect of variable radius into account, the function $h(\epsilon, \tau)$ must be of order $\epsilon^{2}$. Assuming that the field variables $u$, $w$, and $p$ are functions of the slow variables $(\xi, \tau)$ as well as the fast variables $(z, t)$, the following relations hold:

$$
\frac{\partial}{\partial t} \rightarrow \frac{\partial}{\partial t}-\epsilon \lambda \frac{\partial}{\partial \xi}, \quad \frac{\partial}{\partial z} \rightarrow \frac{\partial}{\partial z}+\epsilon \frac{\partial}{\partial \xi}+\epsilon^{2} \frac{\partial}{\partial \tau}
$$

We will further assume that the field variables $u, w, p$ and the function $h(\epsilon, \tau)$ may be expanded into asymptotic series as

$$
\begin{aligned}
& u=\epsilon u_{1}+\epsilon^{2} u_{2}+\epsilon^{3} u_{3}+\cdots, \\
& w=\epsilon w_{1}+\epsilon^{2} w_{2}+\epsilon^{3} w_{3}+\cdots, \\
& p=p_{0}+\epsilon p_{1}+\epsilon^{2} p_{2}+\epsilon^{3} p_{3}+\cdots, \\
& h=\epsilon^{2} h_{1}(\tau)+\epsilon^{3} h_{2}(\tau)+\cdots .
\end{aligned}
$$


Introducing the expansions (3.3) and (3.4) into equations (2.13), (2.14), and (2.16), the following sets of differential equations are obtained.

(i) $O(\epsilon)$-order equations:

$$
\begin{gathered}
2 \frac{\partial u_{1}}{\partial t}+\lambda_{\theta} \frac{\partial w_{1}}{\partial z}=0, \quad \frac{\partial w_{1}}{\partial t}+\frac{\partial p_{1}}{\partial z}=0 \\
p_{1}=\frac{m}{\lambda_{\theta} \lambda_{z}} \frac{\partial^{2} u_{1}}{\partial t^{2}}-\alpha_{0} \frac{\partial^{2} u_{1}}{\partial z^{2}}+\beta_{1} u_{1} .
\end{gathered}
$$

(ii) $O\left(\epsilon^{2}\right)$-order equations:

$$
\begin{gathered}
2 \frac{\partial u_{2}}{\partial t}+\lambda_{\theta} \frac{\partial w_{2}}{\partial z}-2 \lambda \frac{\partial u_{1}}{\partial \xi}+\lambda_{\theta} \frac{\partial w_{1}}{\partial \xi}+u_{1} \frac{\partial w_{1}}{\partial z}=0 \\
\frac{\partial w_{2}}{\partial t}+\frac{\partial p_{2}}{\partial z}-\lambda \frac{\partial w_{1}}{\partial \xi}+\frac{\partial p_{1}}{\partial \xi}+w_{1} \frac{\partial w_{1}}{\partial z}=0 \\
p_{2}=\frac{m}{\lambda_{\theta} \lambda_{z}} \frac{\partial^{2} u_{2}}{\partial t^{2}}-\alpha_{0} \frac{\partial^{2} u_{2}}{\partial z^{2}}+\beta_{1}\left(u_{2}-h_{1}\right) \\
-\frac{2 m \lambda}{\lambda_{\theta} \lambda_{z}} \frac{\partial^{2} u_{1}}{\partial \xi \partial t}-2 \alpha_{0} \frac{\partial^{2} u_{1}}{\partial \xi \partial z}-\frac{m}{\lambda_{\theta}^{2} \lambda_{z}} u_{1} \frac{\partial^{2} u_{1}}{\partial t^{2}} \\
-\alpha_{1}\left(\frac{\partial u_{1}}{\partial z}\right)^{2}-\left(2 \alpha_{1}-\frac{\alpha_{0}}{\lambda_{\theta}}\right) u_{1} \frac{\partial^{2} u_{1}}{\partial z^{2}}+\beta_{2} u_{1}^{2}
\end{gathered}
$$

(iii) $O\left(\epsilon^{3}\right)$-order equations:

$$
\begin{aligned}
& 2 \frac{\partial u_{3}}{\partial t}+\lambda_{\theta} \frac{\partial w_{3}}{\partial z}-2 \lambda \frac{\partial u_{2}}{\partial \xi}+\lambda_{\theta} \frac{\partial w_{2}}{\partial \xi}+w_{1}\left(\frac{\partial u_{1}}{\partial \xi}+\frac{\partial u_{2}}{\partial z}\right)+w_{2} \frac{\partial u_{1}}{\partial z}+\lambda_{\theta} \frac{\partial w_{1}}{\partial \tau}+u_{1}\left(\frac{\partial w_{2}}{\partial z}+\frac{\partial w_{1}}{\partial \xi}\right)+\left(u_{2}-h_{1}\right) \frac{\partial w_{1}}{\partial z}=0 \\
& \frac{\partial w_{3}}{\partial t}+\frac{\partial p_{3}}{\partial z}-\lambda \frac{\partial w_{2}}{\partial \xi}+\frac{\partial p_{2}}{\partial \xi}+\frac{\partial p_{1}}{\partial \tau}+\frac{\partial}{\partial z}\left(w_{1} w_{2}\right)+w_{1} \frac{\partial w_{1}}{\partial \xi}=0 \\
& p_{3}=\frac{m}{\lambda_{\theta} \lambda_{z}} \frac{\partial^{2} u_{3}}{\partial t^{2}}-\alpha_{0} \frac{\partial^{2} u_{3}}{\partial z^{2}}-\frac{2 m \lambda}{\lambda_{\theta} \lambda_{z}} \frac{\partial^{2} u_{2}}{\partial \xi \partial t}-2 \alpha_{0} \frac{\partial^{2} u_{2}}{\partial \xi \partial z} \\
&-\alpha_{0}\left(\frac{\partial^{2} u_{1}}{\partial \xi^{2}}+2 \frac{\partial^{2} u_{1}}{\partial z \partial \tau}\right)+\frac{m \lambda^{2}}{\lambda_{\theta} \lambda_{z}} \frac{\partial^{2} u_{1}}{\partial \xi^{2}}+\beta_{1}\left(u_{3}-h_{2}\right) \\
&-\frac{m}{\lambda_{\theta}^{2} \lambda_{z}} u_{1}\left(\frac{\partial^{2} u_{2}}{\partial t^{2}}-2 \lambda \frac{\partial^{2} u_{1}}{\partial \xi \partial t}\right)-\frac{m}{\lambda_{\theta}^{2} \lambda_{z}}\left(u_{2}-h_{1}\right) \frac{\partial^{2} u_{1}}{\partial t^{2}} \\
&-2 \alpha_{1} \frac{\partial u_{1}}{\partial z}\left(\frac{\partial u_{2}}{\partial z}+\frac{\partial u_{1}}{\partial \xi}\right)-\left(2 \alpha_{1}-\frac{\alpha_{0}}{\lambda_{\theta}}\right) u_{1}\left(\frac{\partial^{2} u_{2}}{\partial z^{2}}+2 \frac{\partial^{2} u_{1}}{\partial z \partial \xi}\right) \\
&-\left(2 \alpha_{1}-\frac{\alpha_{0}}{\lambda_{\theta}}\right)\left(u_{2}-h_{1}\right) \frac{\partial^{2} u_{1}}{\partial z^{2}}+2 \beta_{2} u_{1}\left(u_{2}-h_{1}\right) \\
&+\frac{m}{\lambda_{\theta}^{3} \lambda_{z}} u_{1}^{2} \frac{\partial^{2} u_{1}}{\partial t^{2}}-\left(\alpha_{2}-\frac{\alpha_{1}}{\lambda_{\theta}}\right) u_{1}\left(\frac{\partial u_{1}}{\partial z}\right)^{2} \\
&-\left(\alpha_{2}-\frac{2 \alpha_{1}}{\lambda_{\theta}}+\frac{\alpha_{0}}{\lambda_{\theta}^{2}}\right) u_{1}^{2} \frac{\partial^{2} u_{1}}{\partial z^{2}}-3\left(\gamma_{1}-\frac{\alpha_{0}}{2}\right)\left(\frac{\partial u_{1}}{\partial z}\right)^{2} \frac{\partial^{2} u_{1}}{\partial z^{2}}+\beta_{3} u_{1}^{3}
\end{aligned}
$$




\section{Solution of the field equations}

4.1. The solution of $O(\epsilon)$-order equations. The form of the differential equations (3.5) suggests seeking the following type of solution to these differential equations:

$$
\begin{aligned}
& u_{1}=\left(U_{1} e^{i \theta}+\text { c. с. }\right) \\
& w_{1}=\left(W_{1} e^{i \theta}+\text { c.c. }\right) \\
& p_{1}=\left(-\frac{m \omega^{2}}{\lambda_{\theta} \lambda_{z}}+\alpha_{0} k^{2}+\beta_{1}\right) U_{1} e^{i \theta}+\text { c.c. }
\end{aligned}
$$

where $\omega$ is the angular frequency, $k$ is the wave number, $U_{1}$ and $W_{1}$ are unknown functions of the slow variables $(\xi, \tau), \theta=\omega t-k z$ is the phasor, and c.c. stands for the complex conjugate of the corresponding expressions. Introducing (4.1) into (3.5) we have

$$
U_{1}=U(\xi, \tau), \quad W_{1}=\frac{2}{\lambda_{\theta}} \frac{\omega}{k} U
$$

provided that the following dispersion relation holds true:

$$
\omega^{2}=\frac{\lambda_{\theta}\left(\alpha_{0} k^{2}+\beta_{1}\right) k^{2}}{2+m k^{2} / \lambda_{z}}
$$

Here $U(\xi, \tau)$ is an unknown function whose governing equation will be obtained later.

4.2. Solution of $O\left(\epsilon^{2}\right)$-order equations. Introducing the solution given in (4.1) into (3.6), we have

$$
\begin{gathered}
2 \frac{\partial u_{2}}{\partial t}+\lambda_{\theta} \frac{\partial w_{2}}{\partial z}+2\left(\frac{\omega}{k}-\lambda\right) \frac{\partial U}{\partial \xi} e^{i \theta}-2 i \frac{\omega}{\lambda_{\theta}} U^{2} e^{2 i \theta}+\text { c. c. }=0 \\
\frac{\partial w_{2}}{\partial t}+\frac{\partial p_{2}}{\partial z}+\left(-2 \frac{\lambda}{\lambda_{\theta}} \frac{\omega}{k}-\frac{m \omega^{2}}{\lambda_{\theta} \lambda_{z}}+\alpha_{0} k^{2}+\beta_{1}\right) \frac{\partial U}{\partial \xi} e^{i \theta}-4 i \frac{\omega^{2}}{\lambda_{\theta}^{2} k} U^{2} e^{2 i \theta}+\text { c.c. }=0 \\
p_{2}=\frac{m}{\lambda_{\theta} \lambda_{z}} \frac{\partial^{2} u_{2}}{\partial t^{2}}-\alpha_{0} \frac{\partial^{2} u_{2}}{\partial z^{2}}+\beta_{1} u_{2}+\left[-\beta_{1} h_{1}+2\left(\frac{m \omega^{2}}{\lambda_{\theta}^{2} \lambda_{z}}+\alpha_{1} k^{2}-\frac{\alpha_{0}}{\lambda_{\theta}} k^{2}+\beta_{2}\right)|U|^{2}\right] \\
+2 i\left(\alpha_{0} k-\frac{m \omega \lambda}{\lambda_{\theta} \lambda_{z}}\right) \frac{\partial U}{\partial \xi} e^{i \theta}+\left(\frac{m \omega^{2}}{\lambda_{\theta}^{2} \lambda_{z}}+3 \alpha_{1} k^{2}-\frac{\alpha_{0}}{\lambda_{\theta}} k^{2}+\beta_{2}\right) U^{2} e^{2 i \theta}+\text { c. с. },
\end{gathered}
$$

where $|U|^{2}=U U^{*}, U^{*}$ is the complex conjugate of $U$.

The form of (4.4) suggests seeking the following type of solution:

$$
\begin{aligned}
& u_{2}=U_{2}^{(0)}+\left(\sum_{\ell=1}^{2} U_{2}^{(\ell)} e^{i \ell \theta}+\text { c. c. }\right), \\
& w_{2}=W_{2}^{(0)}+\left(\sum_{\ell=1}^{2} W_{2}^{(\ell)} e^{i \ell \theta}+\text { c. c. }\right) .
\end{aligned}
$$


Introducing (4.5) into (4.4) we obtain

$$
\begin{aligned}
& P_{2}^{(0)}=\beta_{1}\left(U_{2}^{(0)}-h_{1}\right)+2\left(\frac{m \omega^{2}}{\lambda_{\theta}^{2} \lambda_{z}}+\alpha_{1} k^{2}-\frac{\alpha_{0}}{\lambda_{\theta}} k^{2}+\beta_{2}\right)|U|^{2}, \\
& 2 \omega U_{2}^{(1)}-\lambda_{\theta} k W_{2}^{(1)}=2 i\left(\frac{\omega}{k}-\lambda\right) \frac{\partial U}{\partial \xi}, \\
& \omega W_{2}^{(1)}-k\left(-\frac{m \omega^{2}}{\lambda_{\theta} \lambda_{z}}+\alpha_{0} k^{2}+\beta_{1}\right) U_{2}^{(1)} \\
& =i\left(-2 \frac{\lambda}{\lambda_{\theta}} \frac{\omega}{k}-2 m \omega \lambda \frac{k}{\lambda_{\theta} \lambda_{z}}-\frac{m \omega^{2}}{\lambda_{\theta} \lambda_{z}}+3 \alpha_{0} k^{2}+\beta_{1}\right) \frac{\partial U}{\partial \xi}, \\
& 2 \omega U_{2}^{(2)}-\lambda_{\theta} k W_{2}^{(2)}=\frac{\omega}{\lambda_{\theta}} U^{2}, \\
& \omega W_{2}^{(2)}-k\left(-\frac{4 m \omega^{2}}{\lambda_{\theta} \lambda_{z}}+4 \alpha_{0} k^{2}+\beta_{1}\right) U_{2}^{(2)} \\
& =\left(2 \frac{\omega^{2}}{\lambda_{\theta}^{2} k}+\frac{m \omega^{2} k}{\lambda_{\theta}^{2} \lambda_{z}}+3 \alpha_{1} k^{3}-\frac{\alpha_{0}}{\lambda_{\theta}} k^{3}+\beta_{2} k\right) U^{2} .
\end{aligned}
$$

Since, in this work, we will be dealing with the first-order terms in the perturbation expansion, without losing the generality of the problem, we may take $U_{2}^{(1)}=0$. Thus, from (4.7) we obtain

$$
W_{2}^{(1)}=i \frac{2}{\lambda_{\theta} k}\left(\lambda-\frac{\omega}{k}\right) \frac{\partial U}{\partial \xi} \text {. }
$$

Introducing (4.11) into (4.8) we have

$$
\left[\lambda \omega k\left(2+\frac{m k^{2}}{\lambda_{z}}\right)-\left(2 \omega^{2}+\lambda_{\theta} \alpha_{0} k^{4}\right)\right] \frac{\partial U}{\partial \xi}=0
$$

In order to have a nonzero solution for $U$, the coefficient of $\partial U / \partial \xi$ in (4.12) must vanish, that is,

$$
\lambda \omega k\left(2+\frac{m k^{2}}{\lambda_{z}}\right)-\left(2 \omega^{2}+\lambda_{\theta} \alpha_{0} k^{4}\right)=0,
$$

or

$$
\lambda=\frac{2 \omega^{2}+\lambda_{\theta} \alpha_{0} k^{4}}{\omega k\left(2+m k^{2} / \lambda_{z}\right)} \quad \text { (group velocity) }
$$


The solution of (4.9) and (4.10) yields

$$
\begin{aligned}
U_{2}^{(2)} & =\Phi_{0} U^{2}, \quad W_{2}^{(2)}=\frac{2}{\lambda_{\theta}} \frac{\omega}{k} U_{2}^{(2)}-\frac{\omega}{\lambda_{\theta}^{2} k} U^{2}, \\
\Phi_{0} & =\frac{\left(3 \omega^{2}+3 \alpha_{1} \lambda_{\theta} k^{4}-\alpha_{0} k^{4}+\lambda_{\theta} \beta_{2} k^{2}\right)}{3\left(\beta_{1} \lambda_{\theta} k^{2}-2 \omega^{2}\right)} .
\end{aligned}
$$

4.3. Solution of $O\left(\epsilon^{3}\right)$-order equations. For our future purposes, we need the zerothand first-order equations in terms of the phasor $\theta$. For this order of equations we express the variables as

$$
\begin{aligned}
& u_{3}=U_{3}^{(0)}+\left(\sum_{\ell=1}^{3} U_{3}^{(\ell)} e^{i \ell \theta}+\text { c. c. }\right), \\
& w_{3}=W_{3}^{(0)}+\left(\sum_{\ell=1}^{3} W_{3}^{(\ell)} e^{i \ell \theta}+\text { c. c. }\right), \\
& p_{3}=P_{3}^{(0)}+\left(\sum_{\ell=1}^{3} P_{3}^{(\ell)} e^{i \ell \theta}+\text { c. c. }\right) .
\end{aligned}
$$

Introducing the proposed solution (4.16) into (3.7), the zeroth- and the first-order equations may be given as follows:

$$
\begin{gathered}
-2 \lambda \frac{\partial U_{2}^{(0)}}{\partial \xi}+\lambda_{\theta} \frac{\partial W_{2}^{(0)}}{\partial \xi}+\frac{4}{\lambda_{\theta}} \frac{\omega}{k} \frac{\partial}{\partial \xi}|U|^{2}=0 \\
-\lambda \frac{\partial W_{2}^{(0)}}{\partial \xi}+\frac{\partial P_{2}^{(0)}}{\partial \xi}+\frac{4}{\lambda_{\theta}^{2}} \frac{\omega^{2}}{k^{2}} \frac{\partial}{\partial \xi}|U|^{2}=0 \\
2 i \omega U_{3}^{(1)}-i k \lambda_{\theta} W_{3}^{(1)}+\lambda_{\theta} \frac{\partial W_{2}^{(1)}}{\partial \xi}+2 \frac{\omega}{k} \frac{\partial U}{\partial \tau}-2 i \frac{\omega}{\lambda_{\theta}} U_{2}^{(2)} U^{*} \\
-i k W_{2}^{(2)} U^{*}-i\left(k W_{2}^{(0)}+2 \frac{\omega}{\lambda_{\theta}} U_{2}^{(0)}-2 \frac{\omega}{\lambda_{\theta}} h_{1}\right) U=0 \\
\quad i \omega W_{3}^{(1)}-i k P_{3}^{(1)}-\lambda \frac{\partial W_{2}^{(1)}}{\partial \xi}+\frac{\partial P_{2}^{(1)}}{\partial \xi}+\frac{2}{\lambda_{\theta}} \frac{\omega^{2}}{k^{2}} \frac{\partial U}{\partial \tau} \\
P_{3}^{(1)}=\left(\alpha_{0} k^{2}-\frac{m \omega^{2}}{\lambda_{\theta} \lambda_{z}}+\beta_{1}\right) U_{3}^{(1)}+\left(\frac{m \lambda^{2}}{\lambda_{\theta} \lambda_{z}}-\alpha_{0}\right) \frac{\partial^{2} U}{\partial \xi^{2}}+2 i \alpha_{0} k \frac{\partial U}{\partial \tau}-2 i \frac{\omega}{\lambda_{\theta}} W_{2}^{(0)} U=0 \\
+\left(\frac{5 m \omega^{2}}{\lambda_{\theta}^{2} \lambda_{z}}+6 \alpha_{1} k^{2}-5 \frac{\alpha_{0}}{\lambda_{\theta}} k^{2}+2 \beta_{2}\right) U_{2}^{(2)} U^{*} \\
+\left(\frac{m \omega^{2}}{\lambda_{\theta}^{2} \lambda_{z}}+2 \alpha_{1} k^{2}-\frac{\alpha_{0}}{\lambda_{\theta}} k^{2}+2 \beta_{2}\right)\left(U_{2}^{(0)}-h_{1}\right) U \\
+\left(-3 \frac{m \omega^{2}}{\lambda_{\theta}^{3} \lambda_{z}}+2 \alpha_{2} k^{2}-5 \frac{\alpha_{1}}{\lambda_{\theta}} k^{2}+3 \frac{\alpha_{0}}{\lambda_{\theta}^{2}} k^{2}+3\left(\gamma_{1}-\frac{\alpha_{0}}{2}\right) k^{4}+3 \beta_{3}\right)|U|^{2} U
\end{gathered}
$$


The solution of the set (4.17) yields the following result:

$$
\begin{gathered}
U_{2}^{(0)}=\Phi_{1}|U|^{2}-\Phi_{2} h_{1}, \quad W_{2}^{(0)}=2 \frac{\lambda}{\lambda_{\theta}} U_{2}^{(0)}-\frac{4}{\lambda_{\theta}^{2}} \frac{\omega}{k}|U|^{2}, \\
\Phi_{1}=\frac{\left[\left(2 / \lambda_{\theta}\right)(\omega / k)((\omega / k)+\lambda)+m \omega^{2} / \lambda_{\theta} \lambda_{z}+\alpha_{1} \lambda_{\theta} k^{2}-\alpha_{0} k^{2}+\lambda_{\theta} \beta_{2}\right]}{\left(\lambda^{2}-\lambda_{\theta} \beta_{1} / 2\right)}, \\
\Phi_{2}=\frac{\lambda_{\theta} \beta_{1}}{2 \lambda^{2}-\lambda_{\theta} \beta_{1}} .
\end{gathered}
$$

Finally, eliminating $U_{3}^{(1)}, W_{3}^{(1)}$, and $P_{3}^{(1)}$ between (4.18), through the use of the dispersion relation (4.3), the following NLS equation with variable coefficients is obtained:

$$
i \frac{\partial U}{\partial \tau}+\mu_{1} \frac{\partial^{2} U}{\partial \xi^{2}}+\mu_{2}|U|^{2} U-\mu_{3} h_{1}(\tau) U=0,
$$

where the coefficients $\mu_{1}, \mu_{2}$, and $\mu_{3}$ are defined by

$$
\begin{aligned}
\mu_{1}= & {\left[2\left(\alpha_{0} \lambda_{\theta} k^{3}+2 \frac{\omega^{2}}{k}\right)\right]^{-1} } \\
& \times\left[\lambda^{2}\left(2+\frac{m k^{2}}{\lambda_{z}}\right)-4 \frac{\lambda \omega}{k}+2 \frac{m \omega \lambda k}{\lambda_{z}}+\frac{2 \omega^{2}}{k^{2}}-3 \alpha_{0} \lambda_{\theta} k^{2}\right], \\
\mu_{2}= & {\left[2\left(\alpha_{0} \lambda_{\theta} k^{3}+\frac{2 \omega^{2}}{k}\right)\right]^{-1} } \\
& \times\left\{\left[\lambda_{\theta} k^{2}\left(-3 \frac{m \omega^{2}}{\lambda_{\theta}^{3} \lambda_{z}}+2 \alpha_{2} k^{2}-5 \frac{\alpha_{1}}{\lambda_{\theta}} k^{2}+3 \frac{\alpha_{0}}{\lambda_{\theta}^{2}} k^{2}+3\left(\gamma_{1}-\frac{\alpha_{0}}{2}\right) k^{4}+3 \beta_{3}\right)-15 \frac{\omega^{2}}{\lambda_{\theta}^{2}}\right]\right. \\
& +\left[6 \frac{\omega \lambda k}{\lambda_{\theta}}+2 \frac{\omega^{2}}{\lambda_{\theta}}+\lambda_{\theta} k^{2}\left(\frac{m \omega^{2}}{\lambda_{\theta}^{2} \lambda_{z}}+2 \alpha_{1} k^{2}-\frac{\alpha_{0}}{\lambda_{\theta}} k^{2}+2 \beta_{2}\right)\right] \Phi_{1} \\
& \left.+\left[8 \frac{\omega^{2}}{\lambda_{\theta}}+\lambda_{\theta} k^{2}\left(5 \frac{m \omega^{2}}{\lambda_{\theta}^{2} \lambda_{z}}+6 \alpha_{1} k^{2}-5 \frac{\alpha_{0}}{\lambda_{\theta}} k^{2}+2 \beta_{2}\right)\right] \Phi_{0}\right\}, \\
\mu_{3}= & {\left[2\left(\alpha_{0} \lambda_{\theta} k^{3}+\frac{2 \omega^{2}}{k}\right)\right]^{-1} } \\
& \times\left\{2 \frac{\omega^{2}}{\lambda_{\theta}}+\lambda_{\theta} k^{2}\left(\frac{m \omega^{2}}{\lambda_{\theta}^{2} \lambda_{z}}+2 \alpha_{1} k^{2}-\frac{\alpha_{0}}{\lambda_{\theta}} k^{2}+2 \beta_{2}\right)\right. \\
& \left.+\left[6 \frac{\omega \lambda k}{\lambda_{\theta}}+2 \frac{\omega^{2}}{\lambda_{\theta}}+\lambda_{\theta} k^{2}\left(\frac{m \omega^{2}}{\lambda_{\theta}^{2} \lambda_{z}}+2 \alpha_{1} k^{2}-\frac{\alpha_{0}}{\lambda_{\theta}} k^{2}+2 \beta_{2}\right)\right] \Phi_{2}\right\} .
\end{aligned}
$$

Introducing the following change of variable:

$$
U=V(\xi, \tau) \exp \left[-i \mu_{3} \int_{0}^{\tau} h_{1}(s) d s\right],
$$

equation (4.20) reduces to the following conventional NLS equation:

$$
i \frac{\partial V}{\partial \tau}+\mu_{1} \frac{\partial^{2} V}{\partial \xi^{2}}+\mu_{2}|V|^{2} V=0 .
$$


4.4. Progressive wave solution. In this subsection, we will propose the progressive wave solution to (4.23) of the following form:

$$
V(\xi, \tau)=F(\zeta) \exp [i(K \xi-\Omega \tau)], \quad \zeta=\beta\left(\xi-2 \mu_{1} K \tau\right),
$$

where $\Omega, K$, and $\beta$ are some constants and $F(\zeta)$ is a real-valued unknown function to be determined from the solution. Introducing (4.24) into (4.23) we have

$$
\mu_{1} \beta^{2} F^{\prime \prime}+\left(\Omega-\mu_{1} K^{2}\right) F+\mu_{2} F^{3}=0,
$$

where the prime denotes the differentiation of the corresponding quantity with respect to $\zeta$.

As is well known, this equation admits two types of solution depending on the sign of the product $\mu_{1} \mu_{2}$. If $\mu_{1} \mu_{2}>0$, the solution may be given by

$$
F(\zeta)=a \operatorname{sech} \zeta
$$

with

$$
\beta=\left(\frac{\mu_{2}}{2 \mu_{1}}\right)^{1 / 2} a, \quad \Omega=\mu_{1} K^{2}-\frac{\mu_{2}}{2} a^{2} .
$$

If $\mu_{1} \mu_{2}<0$, the solution may be given by

$$
F(\zeta)=a \tanh \zeta
$$

with

$$
\beta=\left(-\frac{\mu_{2}}{2 \mu_{1}}\right)^{1 / 2} a, \quad \Omega=\mu_{1} K^{2}-\mu_{2} a^{2} .
$$

Thus, the solution in terms of $U$ may be given by

$$
U=a \operatorname{sech} \zeta \exp \left[i\left(K \xi-\Omega \tau-\mu_{3} \int_{0}^{\tau} h_{1}(s) d s\right)\right] .
$$

As is seen from this equation, the speed of the enveloping wave is constant and equal to $2 \mu_{1} K$. On the other hand, the speed of the harmonic wave is given by

$$
v_{h}=\frac{\Omega}{K}+\frac{\mu_{3}}{K} h_{1}(\tau)
$$

Here $\Omega / K$ corresponds to the speed of the harmonic wave for the tube of constant radius.

In order to see the effect of stenosis on the harmonic wave speed, one has to know the sign of the coefficient $\mu_{3}$. For this, we must know the constitutive relation of the tube material. In this work, we will utilize the constitutive relation proposed by Demiray [3] for soft biological tissues. Following Demiray [3], the strain energy density function may be expressed as

$$
\Sigma=\frac{1}{2 \alpha}\left\{\exp \left[\alpha\left(\lambda_{\theta}^{2}+\lambda_{z}^{2}+\frac{1}{\lambda_{\theta}^{2} \lambda_{z}^{2}}-3\right)\right]-1\right\},
$$


where $\alpha$ is a material constant and $I_{1}$ is the first invariant of Finger deformation tensor and defined by $I_{1}=\lambda_{\theta}^{2}+\lambda_{z}^{2}+1 / \lambda_{\theta}^{2} \lambda_{z}^{2}$. Introducing (4.32) into (2.18), the explicit expressions of the coefficients $\alpha_{0}, \alpha_{1}, \beta_{1}$, and $\beta_{2}$ may be given as follows:

$$
\begin{aligned}
& \alpha_{0}=\frac{1}{\lambda_{\theta}}\left(\lambda_{z}-\frac{1}{\lambda_{\theta}^{2} \lambda_{z}^{3}}\right) G\left(\lambda_{\theta}, \lambda_{z}\right), \\
& \alpha_{1}=\left[\frac{1}{\lambda_{\theta}^{4} \lambda_{z}^{3}}+\alpha\left(\lambda_{z}-\frac{1}{\lambda_{\theta}^{2} \lambda_{z}^{3}}\right)\left(1-\frac{1}{\lambda_{\theta}^{4} \lambda_{z}^{2}}\right)\right] G\left(\lambda_{\theta}, \lambda_{z}\right), \\
& \beta_{1}=\left[\frac{4}{\lambda_{\theta}^{5} \lambda_{z}^{3}}+2 \frac{\alpha}{\lambda_{\theta} \lambda_{z}}\left(\lambda_{\theta}-\frac{1}{\lambda_{\theta}^{3} \lambda_{z}^{2}}\right)^{2}\right] G\left(\lambda_{\theta}, \lambda_{z}\right), \\
& \beta_{2}=\left[-\frac{10}{\lambda_{\theta}^{6} \lambda_{z}^{3}}+\frac{\alpha}{\lambda_{\theta} \lambda_{z}}\left(\lambda_{\theta}-\frac{1}{\lambda_{\theta}^{3} \lambda_{z}^{2}}\right)\left(1+\frac{11}{\lambda_{\theta}^{4} \lambda_{z}^{2}}\right)+2 \frac{\alpha^{2}}{\lambda_{\theta} \lambda_{z}}\left(\lambda_{\theta}-\frac{1}{\lambda_{\theta}^{3} \lambda_{z}^{2}}\right)^{3}\right] G\left(\lambda_{\theta}, \lambda_{z}\right),
\end{aligned}
$$

where the function $G$ is defined by

$$
G\left(\lambda_{\theta}, \lambda_{\theta}\right)=\exp \left[\alpha\left(\lambda_{\theta}^{2}+\lambda_{z}^{2}+\frac{1}{\lambda_{\theta}^{2} \lambda_{z}^{2}}-3\right)\right] .
$$

In order to study the variation of the coefficient $\mu_{3}$ with the initial deformation, we need the value of material constant $\alpha$. For the static case, the present model was compared by Demiray [4] with the experimental measurements by Simon et al. [16] on canine abdominal artery with the characteristics $R_{i}=0.31 \mathrm{~cm}, R_{0}=0.38 \mathrm{~cm}$, and $\lambda_{z}=1.53$, and the value of the material constant $\alpha$ was found to be $\alpha=1.948$. The numerical analysis of the coefficient $\mu_{3}$ as a function of $\lambda_{\theta}, \lambda_{z}$, and the wave number $k$ indicates that the coefficient $\mu_{3}$ is always negative. This means that when $h_{1}(\tau)$ is a decreasing function of the distance parameter $\tau$, the wave speed increases with the distance from the center of the stenosis. Such a result is to be expected from physical considerations.

ACKNOWLEDGMENT. This work was supported by the Turkish Academy of Sciences (TÜBA).

\section{REFERENCES}

[1] M. Anliker, R. L. Rockwell, and E. Ogden, Nonlinear analysis of flow pulses and shock waves in arteries, Z. Angew. Math. Phys. 22 (1968), 217-246.

[2] H. B. Atabek and H. S. Lew, Wave propagation through a viscous incompressible fluid contained in an initially stressed elastic tube, Biophys. J. 7 (1966), 486-503.

[3] H. Demiray, On the elasticity of soft biological tissues, J. Biomech. 5 (1972), 309-311.

[4] _ Large deformation analysis of some basic problems in biophysics, Bull. Math. Biol. 38 (1976), 701-712.

[5] _ Wave propagation through a viscous fluid contained in a prestressed thin elastic tube, Internat. J. Engrg. Sci. 30 (1992), no. 11, 1607-1620.

[6] _ Solitary waves in prestressed elastic tubes, Bull. Math. Biology 58 (1996), no. 5, 939-955.

[7] _ Modulation of non-linear waves in a viscous fluid contained in an elastic tube, Int. J. Nonlinear Mech. 36 (2001), 649-661. 
[8] Y. C. Fung, Biodynamics: Circulation, Springer, New York, 1981.

[9] Y. Hazhizume, Nonlinear pressure waves in a fluid-filled elastic tube, J. Phys. Soc. Japan 54 (1985), 3305-3312.

[10] R. S. Johnson, A nonlinear equation incorporating damping and dispersion, J. Fluid Mech. 42 (1970), 49-60.

[11] S. C. Ling and H. B. Atabek, A nonlinear analysis of pulsatile blood flow in arteries, J. Fluid Mech. 55 (1972), 492-511.

[12] T. J. Pedley, The Fluid Mechanics of Large Blood Vessels, Cambridge University Press, Cambridge, 1980.

[13] A. J. Rachev, Effects of transmural pressure and muscular activity on pulse waves in arteries, J. Biomech. Eng. 102 (1980), 119-123.

[14] R. Ravindran and P. Prasad, A mathematical analysis of nonlinear waves in a fluid-filled viscoelastic tube, Acta Mech. 31 (1979), 253-280.

[15] G. Rudinger, Shock waves in a mathematical model of aorta, J. Appl. Mech. 37 (1970), 34-37.

[16] B. R. Simon, A. S. Kobayashi, D. E. Stradness, and C. A. Wiederhielm, Re-evaluation of arterial constitutive laws, Circ. Res. 30 (1972), 491-500.

[17] R. J. Tait and T. B. Moodie, Waves in nonlinear fluid filled tubes, Wave Motion 6 (1984), 197-203.

[18] Y. Yomosa, Solitary waves in large blood vessels, J. Phys. Soc. Japan 56 (1987), 506-520.

Hilmi Demiray: Department of Mathematics, Faculty of Arts and Sciences, Isik University, Büyükdere Caddesi, 34398 Maslak, Istanbul, Turkey

E-mail address: demi ray@isikun.edu.tr 


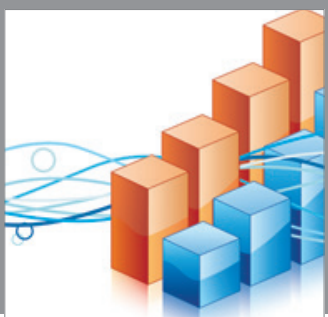

Advances in

Operations Research

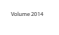

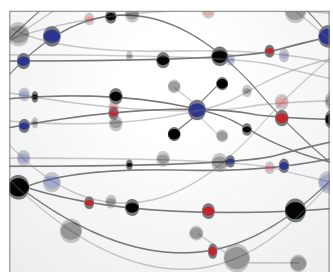

\section{The Scientific} World Journal
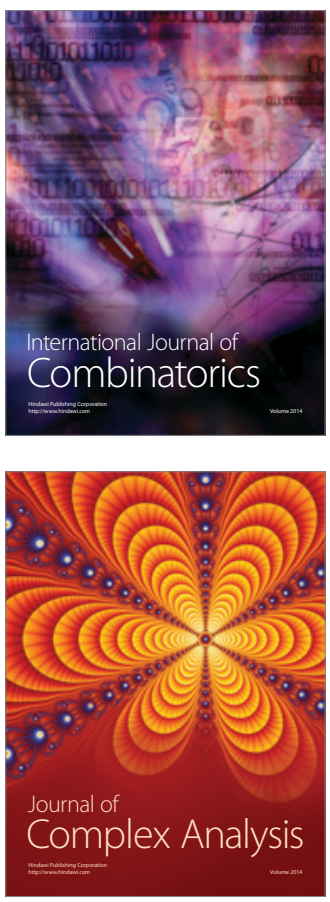

International Journal of

Mathematics and

Mathematical

Sciences
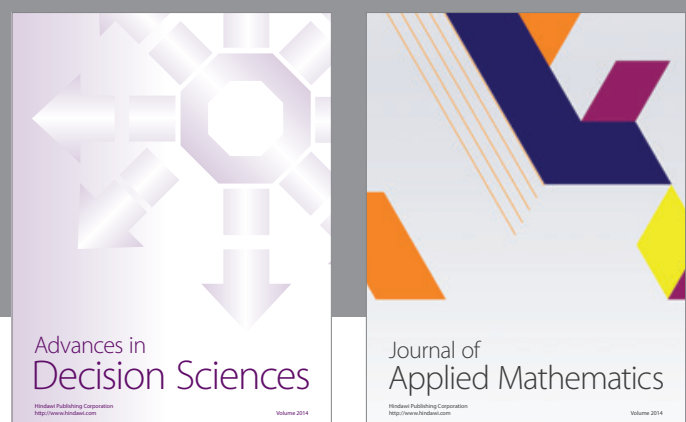

Journal of

Applied Mathematics
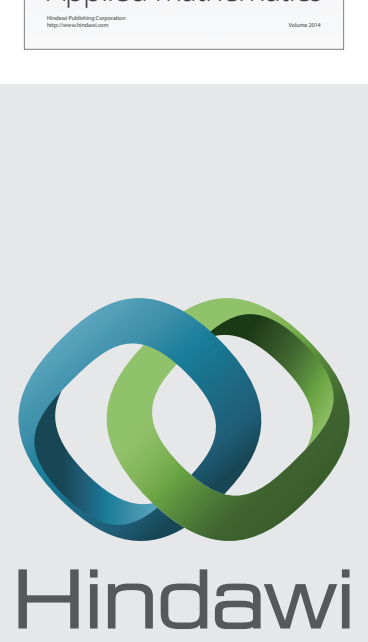

Submit your manuscripts at http://www.hindawi.com
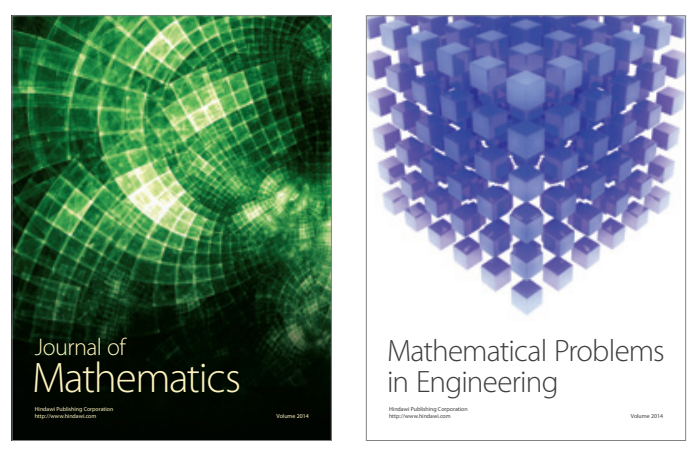

Mathematical Problems in Engineering
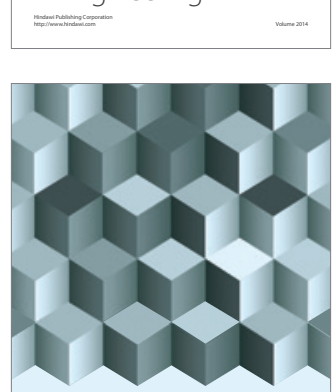

Journal of

Function Spaces
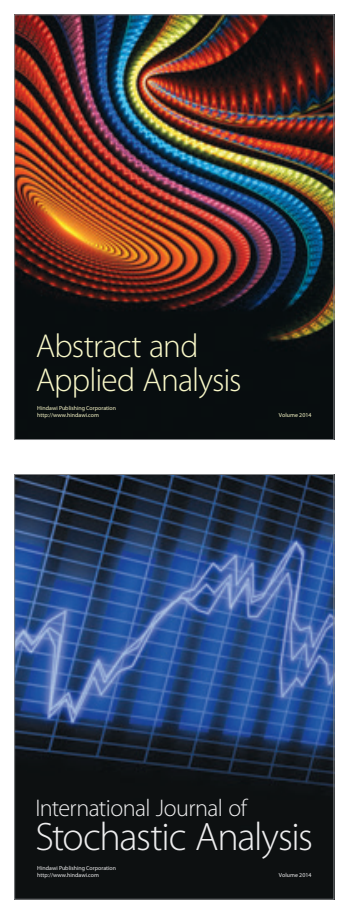

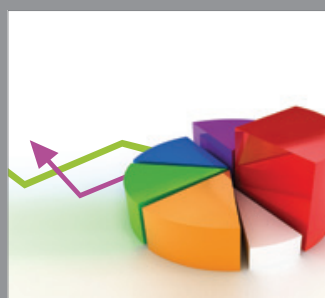

ournal of

Probability and Statistics

Promensencen
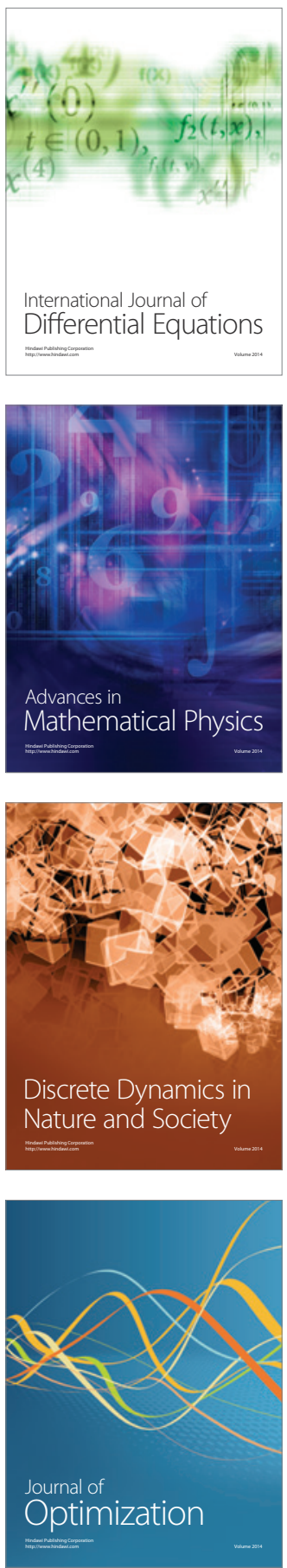